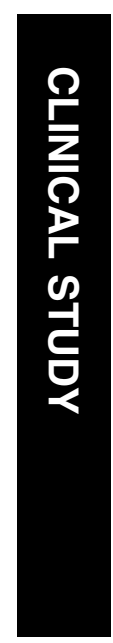

\title{
Causes of blind and partial sight certifications in England and Wales: April 2007-March 2008
}

Research and Development, Moorfields Eye Hospital, London, UK

Correspondence: C Bunce, Research and Development, Moorfields Eye Hospital, City Road, London EC1V 2PD, UK

Tel: +44 207566 2820;

Fax: +442086086925.

E-mail: c.bunce@ucl.ac.uk

Received: 27 January 2010 Accepted in revised form:

4 July 2010

Published online:

17 September 2010

\begin{abstract}
Purpose The last complete report on causes of blindness in England and Wales was for the data collected during April 1999-March 2000. This study updates these figures, with data collected during April 2007-March 2008. Methods In England and Wales, registration for blindness and partial sight is initiated with certification by a consultant ophthalmologist with the consent of the patient. The main cause of visual impairment was ascertained where possible for all certificates completed during April 2007-March 2008 and a proportional comparison with 1999-2000 figures was made.

Results We received 23185 Certificates of Vision Impairment (CVIs), of which 9823 were for severe sight impairment (blindness) (SSI) and 12607 were for sight impairment (partial sight) (SI). These totals were considerably lower than the numbers certified in the year ending 31 March 2000. In 16.6\% of CVIs, there were multiple causes of visual impairment as compared with 3\% of BD8s in 2000.

Degeneration of the macula and posterior pole (mostly age-related macular degeneration (AMD)) contributed to vision impairment in 12746 newly certified blind or partially sighted. Conclusions AMD is still by far the leading cause of certified visual loss in England and Wales. Proportional comparisons are hampered by the increasing use of multiple pathology as a main cause of visual impairment, which is believed to have arisen owing to the change in certificate used for data collection. These figures are not estimates of the total numbers newly blind in the UK because not all those entitled to certification are offered and or accept it, but they do nevertheless document the number of people
\end{abstract}

C Bunce, W Xing and R Wormald

who are deemed to be sufficiently sight impaired to warrant support and have been both offered and accepted it. This is usually the case when no further ophthalmic intervention is thought likely to be of benefit in terms of restoring or improving vision. Eye (2010) 24, 1692-1699; doi:10.1038/eye.2010.122; published online 17 September 2010

Keywords: blind; registration; age-related macular degeneration; glaucoma; diabetic retinopathy

\section{Introduction}

The number of blind people in Britain has been counted since 1851, and reports on the causes of low vision in England and Wales began in 1950. ${ }^{1-6}$ From the mid-1930s, registration as blind or partially sighted in England and Wales was initiated by completion of a designated certificate - the BD8, which required the signature of an ophthalmologist. Part 5 of the BD8 was an anonymous epidemiological return containing data on the cause of visual impairment, which was sent for analysis to the Office of Population Censuses and Surveys (OPCS) - now known as the Office of National Statistics (ONS). ${ }^{7-9}$ In September 2005, the BD8 was replaced in England by the Certificate of Vision Impairment (CVI), with copies of the form being sent for epidemiological analysis to the Certifications Office, London. In April 2007, Wales released its equivalent form - the CVI-W. This is a report of an analysis conducted on all CVIs and CVI-Ws, with certification dates between 1 April 2007 and 31 March 2008, which arrived at the Certifications Office London before November 2008 (at which point the dataset was locked). 
Table 1 Main causes of severe sight impairment (blindness): certifications April 2007-March 2008

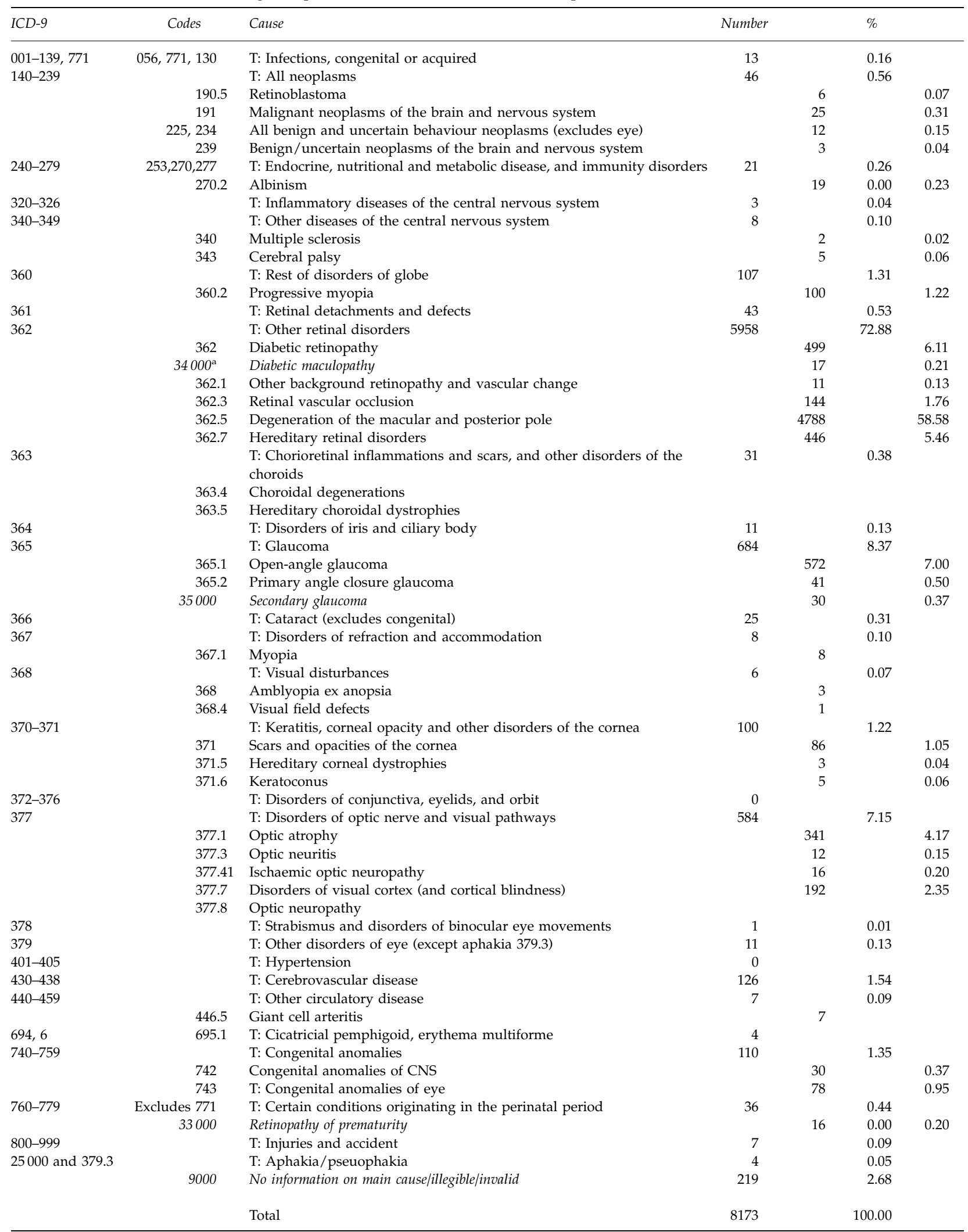

Abbreviation: $\mathrm{T}$, total.

${ }^{a}$ Codes in italics are not ICD-9 codes, but were created for the CVI analysis. 
Table 2 Main causes of sight impairment (partial sight): certifications April 2007-March 2008

\begin{tabular}{|c|c|c|c|c|c|c|}
\hline$I C D-9$ & Codes & Cause & Number & & $\%$ & \\
\hline 001-139, 771 & $042,771,130$ & T: Infections, congenital or acquired & 5 & & 0.05 & \\
\hline \multirow[t]{6}{*}{$140-239$} & & T: All neoplasms & 64 & & 0.61 & \\
\hline & 190.5 & Retinoblastoma & & 3 & & 0.03 \\
\hline & 191 & Malignant neoplasms of the brain and nervous system & & 47 & & 0.45 \\
\hline & 194 & Malignant neoplasms of pituitary gland & & 3 & & 0.03 \\
\hline & 225,234 & All benign and uncertain behaviour neoplasms (excludes eye) & & 8 & & 0.08 \\
\hline & 239 & Benign/uncertain neoplasms of the brain and nervous system & & 3 & & 0.03 \\
\hline \multirow[t]{2}{*}{ 240-279 } & & T: Endocrine, nutritional and metabolic disease, and immunity disorders & 70 & & 0.67 & \\
\hline & 270.2 & Albinism & & 70 & & 0.67 \\
\hline $320-326$ & & $\mathrm{~T}$ : Inflammatory diseases of the central nervous system & 2 & & 0.02 & \\
\hline 330-337 & 330,333 & $\mathrm{~T}$ : Hereditary and degenerative diseases of the CNS & 5 & & 0.05 & \\
\hline \multirow[t]{3}{*}{$340-349$} & & T: Other diseases of the central nervous system & 9 & & 0.09 & \\
\hline & 340 & Multiple sclerosis & & 2 & & 0.02 \\
\hline & 343 & Cerebral palsy & & 1 & & 0.01 \\
\hline \multirow[t]{2}{*}{360} & & T: Rest of disorders of globe & 143 & & 1.36 & \\
\hline & 360.2 & Progressive myopia & & 140 & & 1.32 \\
\hline 361 & & T: Retinal detachments and defects & 39 & & 0.37 & \\
\hline \multirow[t]{7}{*}{362} & & $\mathrm{~T}$ : Other retinal disorders & 7452 & & 71.01 & \\
\hline & 362 & Diabetic retinopathy & & 765 & & 7.29 \\
\hline & $34000^{\mathrm{a}}$ & Diabetic maculopathy & & 30 & & 0.29 \\
\hline & 362.1 & Other background retinopathy and vascular change & & 15 & & 0.14 \\
\hline & 362.3 & Retinal vascular occlusion & & 126 & & 1.20 \\
\hline & 362.5 & Degeneration of the macular and posterior pole & & 6004 & & 57.21 \\
\hline & 362.7 & Hereditary retinal disorders & & 443 & & 4.22 \\
\hline \multirow[t]{3}{*}{363} & & T: Chorioretinal inflammations and scars, and other disorders of the choroids & 34 & & 0.32 & \\
\hline & 363.4 & Choroidal degenerations & & 11 & & 0.10 \\
\hline & 363.5 & Hereditary choroidal dystrophies & & 5 & & 0.05 \\
\hline 364 & & $\mathrm{~T}$ : Disorders of iris and ciliary body & 15 & & 0.14 & \\
\hline \multirow[t]{4}{*}{365} & & T: Glaucoma & 772 & & 7.36 & \\
\hline & 365.1 & Open-angle glaucoma & & 683 & & 6.51 \\
\hline & 365.2 & Closed-angle glaucoma & & 28 & & 0.27 \\
\hline & 35000 & Secondary glaucoma & & 26 & & 0.25 \\
\hline 366 & & T: Cataract (excludes congenital) & 31 & & 0.30 & \\
\hline \multirow[t]{2}{*}{367} & & T: Disorders of refraction and accommodation & 7 & & 0.07 & \\
\hline & 367.1 & Myopia & & & & \\
\hline \multirow[t]{3}{*}{368} & & $\mathrm{~T}$ : Visual disturbances & 41 & & 0.39 & \\
\hline & 368 & Amblyopia ex anopsia & & 23 & & 0.22 \\
\hline & 368.4 & Visual field defects & & & & \\
\hline \multirow[t]{4}{*}{ 370-371 } & & T: Keratitis, corneal opacity, and other disorders of the cornea & 130 & & 1.24 & \\
\hline & 371 & Scars and opacities of the cornea & & 100 & & 0.95 \\
\hline & 371.5 & Hereditary corneal dystrophies & & 7 & & 0.07 \\
\hline & 371.6 & Keratoconus & & 11 & & 0.10 \\
\hline 372-376 & 376 & T: Disorders of conjunctiva, eyelids, and orbit & 1 & & 0.01 & \\
\hline \multirow{6}{*}{377} & & T: Disorders of optic nerve and visual pathways & 651 & & 6.20 & \\
\hline & 377.1 & Optic atrophy & & 308 & & 2.93 \\
\hline & 377.3 & Optic neuritis & & 12 & & 0.11 \\
\hline & 377.41 & Ischaemic optic neuropathy & & 8 & & 0.08 \\
\hline & 377.7 & Disorders of visual cortex (and cortical blindness) & & 304 & & 2.90 \\
\hline & 377.8 & Optic neuropathy & & & & \\
\hline 378 & & T: Strabismus and disorders of binocular eye movements & 2 & & 0.02 & \\
\hline \multirow[t]{2}{*}{379} & & T: Other disorders of eye (except aphakia 379.3) & 88 & & 0.84 & \\
\hline & 379.5 & Nystagmus & & 87 & & 0.83 \\
\hline $430-438$ & & $\mathrm{~T}$ : Cerebrovascular disease & 454 & & 4.33 & \\
\hline \multirow[t]{2}{*}{$440-459$} & & T: Other circulatory disease & 2 & & 0.02 & \\
\hline & 446.5 & Giant cell arteritis & & 2 & & 0.02 \\
\hline \multirow[t]{3}{*}{$740-759$} & & T: Congenital anomalies & 141 & & & \\
\hline & 742 & Congenital anomalies of the CNS & & 34 & & 0.32 \\
\hline & 743 & T: Congenital anomalies of the eye & & 104 & & 0.99 \\
\hline 760-779 & Excludes 771 & $\mathrm{~T}$ : Certain conditions originating in the perinatal period & 27 & & 0.26 & \\
\hline & 33000 & Retinopathy of prematurity & & 12 & & 0.11 \\
\hline 800-999 & & $\mathrm{T}$ : Injuries and accident & 20 & & 0.19 & \\
\hline & $9000^{\mathrm{a}}$ & No information on main cause/illegible/invalid & 288 & & 2.74 & \\
\hline & & Total & 10495 & & 100.00 & \\
\hline
\end{tabular}

Abbreviation: $\mathrm{T}$, total.

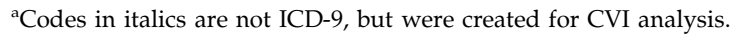




\section{Materials and methods}

An electronic version of the CVI, the ECVI, was used at the Certifications Office to transfer information from the paperbased certificates into a database. Data were coded using ICD-9 for ready comparison with the analysis conducted on the 1999-2000 data - the last causal analysis conducted. ${ }^{10}$

Data entry for $5 \%$ of the forms was duplicated to check for the frequency of transcription and coding errors. Duplicate forms were then removed from the dataset.

Part C of the CVI form collects information on the cause of visual loss. It contains a picking list of common diagnoses and instructs the person completing the form to select main cause using an asterisk or circle. Guidelines are provided in the 'explanatory notes for consultant ophthalmologists' as to how to select a single main cause of visual loss where this is not evident.

Previous recent analyses on certification data were conducted on the main cause of blindness or partial sight. To facilitate comparison with these analyses and to provide information for ophthalmologists of varying specialties, we tabulated the number of certificates attributed to each main cause of visual impairment and

Table 3 Prevalence of multiple causes in certifiable vision impairment by age group and certification status: certifications April 2007-March 2008

\begin{tabular}{lcrrrrr}
\hline Study factor & & \multicolumn{2}{c}{ Single cause } & & \multicolumn{2}{c}{ Multiple cause } \\
\cline { 3 - 4 } \cline { 7 - 7 } & & Number & Row \% & & Number & Row \% \\
\hline Age at & $0-15$ & 849 & 85.4 & & 145 & 14.6 \\
certification & $16-64$ & 2968 & 85.0 & & 523 & 15.0 \\
& $65-74$ & 2088 & 82.4 & & 445 & 17.6 \\
& $75-84$ & 6028 & 83.0 & & 1230 & 16.9 \\
& 85 plus & 6291 & 82.1 & & 1369 & 17.9 \\
Certification & SSI & 8173 & 83.2 & & 1650 & 16.8 \\
Status & SI & 10497 & 83.3 & & 2110 & 16.7 \\
\hline
\end{tabular}

show these numbers as percentages of the total number of certificates for blindness/partial sight (Tables 1 and 2).

For the CVI dataset however, the proportion of forms with the main cause of visual loss being recorded as 'multiple' cause was significantly higher than was the case for the BD8 forms, which were used for the 1999 report (16.6\% vs just over 3\%). Multiple causes are used where the ophthalmologist has not indicated a single cause of visual loss - there may be differing causes in the two eyes or more than one cause within one eye and the ophthalmologist is unable to decide which contributes most to certifiable visual loss. While the BD8 prompted the ophthalmologist to enter a main cause using a single large text field on the form, the instructions on the CVI are less obvious, and while guidance is provided in the explanatory notes, these are unlikely to be readily available for the busy consultant in clinic. We tabulated multiple cause against age and visual status to assess whether or not this influenced the likelihood of multiple cause being recorded (Table 3 ).

Tables 4 and 5 presents figures for leading causes, including the number of certificates where this was the main cause of visual impairment and where a multiple cause had been recorded and that condition was a contributory diagnosis. This means that a certificate can contribute to more than one cause.

\section{Results}

We received 23185 CVI certificates dated between April 2007 and March 2008, of which 9823 were people certified with severe sight impairment (blindness) (SSI) and 12607 certified with sight impairment (partial sight) (SI). An additional 755 (3.3\%) forms did not state whether or not the individual was SSI or SI-this compares with 1515 (4.4\%) of 34410 BD8s completed during the period April 1999-March 2000.

Table 4 Numbers of SSI (blindness) CVIs by cause, with that cause as the main cause of CVI or with the main cause recorded as multiple, but a contributory cause being that condition: certifications April 2007-March 2008

\begin{tabular}{|c|c|c|c|c|}
\hline ICD-9 & Diagnosis & Single cause & Contributory cause & Total \\
\hline 362.5 & Degeneration macular and posterior pole & 4788 & 916 & 5704 \\
\hline 365 & Glaucoma & 684 & 719 & 1403 \\
\hline $362 / 34000$ & Diabetic retinopathy/maculopathy & 516 & 138 & 654 \\
\hline 362.7 & Hereditary retinal disorders & 446 & 129 & 575 \\
\hline 377.1 & Optic atrophy & 341 & 164 & 505 \\
\hline 377.7 & Disorders of visual cortex & 192 & 94 & 286 \\
\hline 362.3 & Retinal vascular occlusion & 144 & 243 & 387 \\
\hline $430-438$ & Cerebrovascular disease & 126 & 111 & 237 \\
\hline 360.2 & Myopia & 100 & 95 & 196 \\
\hline \multirow[t]{2}{*}{$370-371$} & Keratitis, corneal opacity, and other disorders of the cornea & 100 & 150 & 250 \\
\hline & & 7437 & 2759 & 10196 \\
\hline
\end{tabular}

Abbreviation: CVI, certifiable visual loss. 
Table 5 Numbers of SI (partial sight) CVIs by cause, with that cause as the main cause of CVI or with a main cause recorded as multiple, but a contributory cause being that condition

\begin{tabular}{|c|c|c|c|c|}
\hline$I C D-9$ & Diagnosis & Single cause & Contributory cause & Total \\
\hline 362.5 & Degeneration macular and posterior pole & 6004 & 1038 & 7042 \\
\hline 365 & Glaucoma & 772 & 831 & 1603 \\
\hline $362 / 34000$ & Diabetic retinopathy/maculopathy & 795 & 208 & 1003 \\
\hline 362.7 & Hereditary retinal disorders & 443 & 140 & 583 \\
\hline 377.1 & Optic atrophy & 308 & 181 & 489 \\
\hline 377.7 & Disorders of visual cortex & 304 & 145 & 449 \\
\hline 362.3 & Retinal vascular occlusion & 126 & 335 & 461 \\
\hline $430-438$ & Cerebrovascular disease & 454 & 212 & 666 \\
\hline 360.2 & Malignant myopia & 140 & 100 & 240 \\
\hline \multirow[t]{2}{*}{$370-371$} & Keratitis, corneal opacity, and other disorders of the cornea & 130 & 179 & 309 \\
\hline & & 9553 & 3393 & 12945 \\
\hline
\end{tabular}

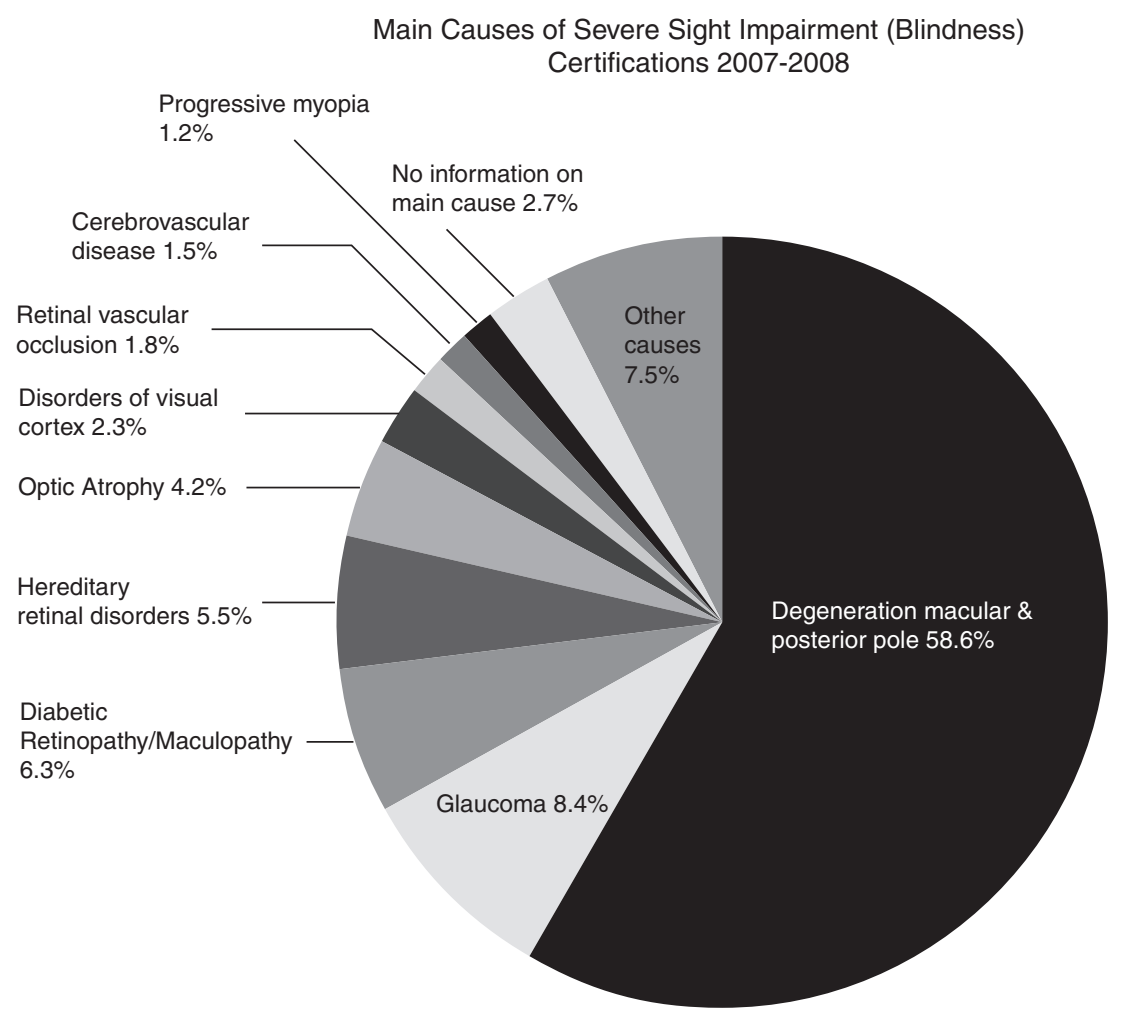

Figure 1 Main causes of severe sight impairment (blindness) in England and Wales: certifications April 2007-March 2008.

The main causes of certifiable SSI are shown in Table 1, columns 4 and 5 show the number of certificates attributed to each cause and columns 6 and 7 show these numbers as percentages of the total number of certificates for SSI.

Figure 1 shows the relative percentages of the leading causes of certifiable SSI. The most commonly recorded main cause of certification for SSI was degeneration of the macula and posterior pole (ICD 362.5) (58.6\%), which largely comprises age-related macula degeneration. This was similar to what was seen in 1999-2000 where the figure for age-related macular degeneration (AMD) was $57.2 \%$. As in 1999-2000, glaucoma (8.4\%) and diabetic retinopathy $(6.3 \%)$ were the next frequently occurring causes of certification for SSI, where there was a single cause. However, while in 1999-2000, hereditary retinal disorders were responsible for $2.8 \%$ of certifications for SSI, in 2007-2008, this figure has risen to 5.5\%. Optic 
Main Causes of Sight Impairment (Partial Sight)

\section{Certifications 2007-2008}

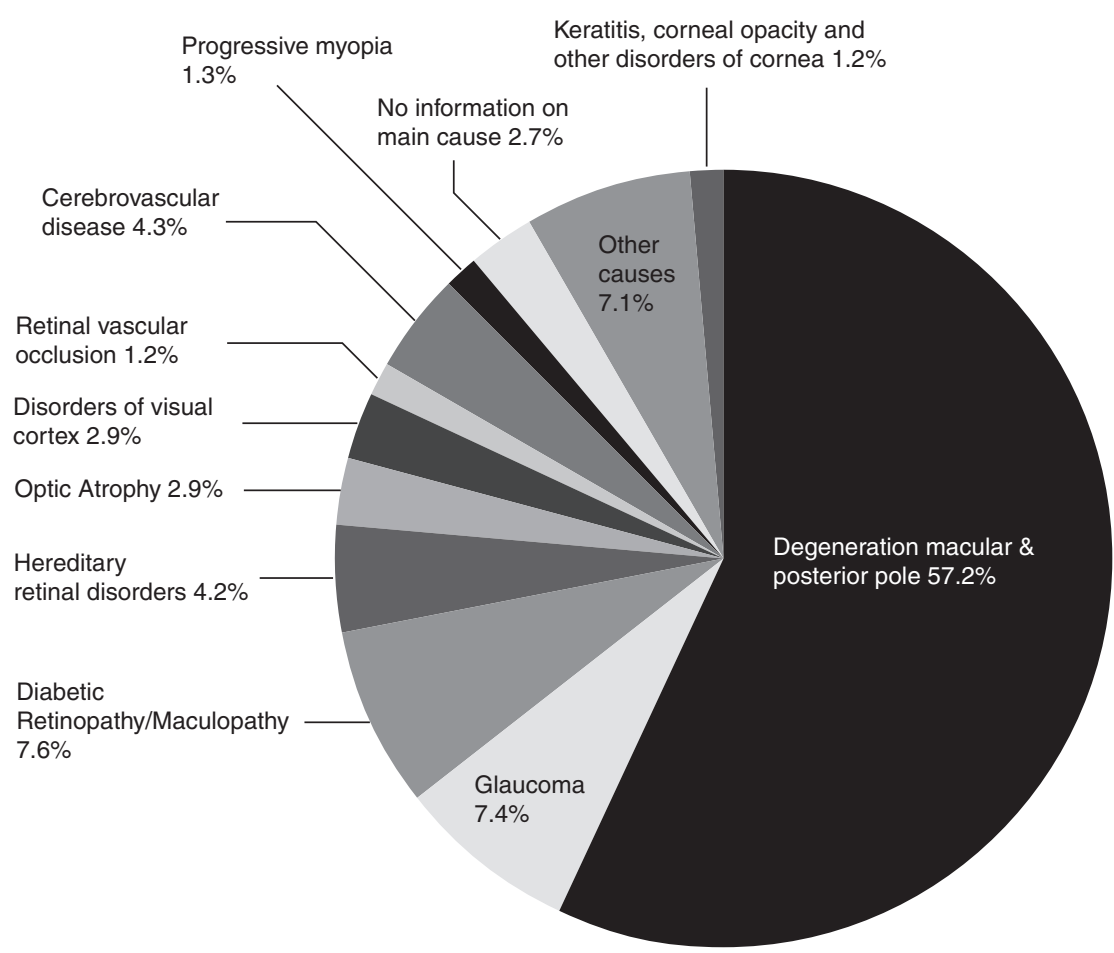

Figure 2 Main causes of sight impairment (partial sight) in England and Wales: certifications April 2007-March 2008.

atrophy (4.2\%) and disorders of the visual cortex (2.3\%) were the next frequently occurring causes of certification for SSI. Taken together, these accounted for over $80 \%$ of SSI certifications during the year.

The main causes of certifiable SI are shown in Table 2, columns 4 and 5 show the number of certificates attributed to each cause and columns 6 and 7 show these numbers as percentages of the total number of certificates for SI.

Figure 2 shows the relative percentages of the causes of SI certification. As for SSI, the most commonly recorded main cause of certification for SI was degeneration of the macula and posterior pole (57.2\%). Diabetic eye disease $(7.6 \%)$, glaucoma $(7.4 \%)$, cerebrovascular disease $(4.3 \%)$, hereditary retinal disorders $(4.2 \%)$, and optic atrophy $(2.9 \%)$ were frequently occurring causes of certification for SI, as for SSI.

There were 3859 forms received, which did not specify a main cause of certification, of these 1607 (41.6\%) had a different cause in the right and left eye, and 2252 (58.4\%) had more than one cause in at least one eye. Table 3 suggests that use of multiple cause was slightly more common in the older age groups, but was similar in SI and SSI certificates.
Tables 4 and 5 present leading causes of certifiable sight impairment based both on single cause forms and multiple case forms. As multiple pathology featured significantly more often in CVI data than BD8 data and because we believe that this is likely to reflect the change in mechanism for data capture, rather than a change in incident vision impairment, these figures should be adopted when comparing crude numbers by cause.

\section{Discussion}

The aim of this paper is to provide updated figures on causes of certifiable vision impairment in England and Wales and to highlight some of the issues that need to be addressed when comparing current crude estimates with previous years.

To facilitate comparison with the 1999-2000 data, we have presented the number of certificates attributed to each main cause of vision impairment and shown these numbers as percentages of the total number of certificates for SSI and SI. There are similarities in this analysis and the previous. AMD is by far the leading cause of all-ages certifications for SSI and SI. In 1999-2000, this condition 
accounted for $57.2 \%$ of certifications for SSI, whereas in 2007-2008, it accounted for 58.6\%. Unfortunately, neither the ICD-9 nor the ICD-10 coding classifications make the distinction between neovascular and geographic AMD. The CVI form does however, and work is currently underway at the Certifications Office so that figures by AMD type can be provided. As in 1999-2000, glaucoma $(8.4 \%)$ and diabetic retinopathy $(6.2 \%)$ were the next frequently occurring causes of certification for SSI. Changes are also seen however, with an increase in the proportion of forms attributed to hereditary retinal disorders. Proportionately, glaucoma has decreased slightly over the time period for SSI (10.9 to 8.4\%) and for SI $(10.2$ to $7.4 \%)$.

It is important to note that, however, a proportional comparison requires caution, as an increase in one cause can result in a proportionate decrease in another cause.

This proportional comparison is further complicated by the increase in the proportion of forms where a main cause has not been identified. In the 1999-2000 dataset, which was derived from BD8s, approximately $3 \%$ of forms had a 'multiple cause'; in the 2007-2008 dataset, this has increased to $16.6 \%$. There appeared to be a slight increase in the use of multiple causes with age, but the proportions of SI and SSI CVIs with multiple causes were similar.

As a result of this, we believe that when comparing numbers attributed to each cause over time, the figures given in Tables 4 and 5 should be cited. Temporal comparison of data is the focus of a future paper, but we feel it of value to put these figures in the public domain so that the ophthalmologists who complete the forms can see the value of their data. What cannot be overlooked, however, when making temporal comparisons, is the fact that the numbers being registered blind and partially sighted has significantly decreased and that this drop coincided with the launch of the new form. While this might reflect decreases in the numbers with newly incident blindness, anecdotal data and epidemiological modeling suggest that these numbers should be increasing. The numbers of certificates are significantly lower than were seen in 1999-2000, when 34410 certificates were received -13788 people were newly certified blind and 19107 people were newly certified partially sighted. The numbers of certificates are also lower than the figures reported as registered at Social Services during the year ending 2006 when 10820 subjects were registered blind and 14375 were registered partially sighted. ${ }^{11}$ It would appear therefore that certification and registration figures have fallen considerably over the last 10 years. The NHS Information Centre for health and social care have studied changes in registration figures between 2003, 2006, and 2008, and have found that blind and partial sight registrations have been decreasing in most age groups, yet have increased in the 17 and under age category (and for the 18-49 age group for partial sight).

Under-certification was reported as an issue for BD8 certificates and it appears that this is likely to be occurring with CVIs also. ${ }^{12-17}$ It is almost certainly true that many people who are eligible for certification are not certified and that many people who are certified may not always satisfy the criteria for certification. These reasons are often used to devalue these data for epidemiological purposes, but we believe that it is still useful to have knowledge of the frequency and distribution of eye conditions, which cause sight loss of sufficient severity to warrant the offer and acceptance of registration. People who have lost vision are in need of support, and without a certification system, it is difficult to see how support will be activated or how their numbers can be monitored.

The CVI, like the BD8, was not piloted before being introduced, but with a move in the NHS to increasing use of IT, it is hoped that the whole process could be managed electronically with forced choice diagnostic categories and automated coding. The Certifications Office makes use of an electronic version of the CVI, which was developed as part of a project funded by Guide Dogs. Widespread implementation of this technology across the NHS will improve data collection and allow users to have a greater role in determining the contents of the CVI. We encourage people to continue completing the CVI and to adopt the electronic CVI where possible.

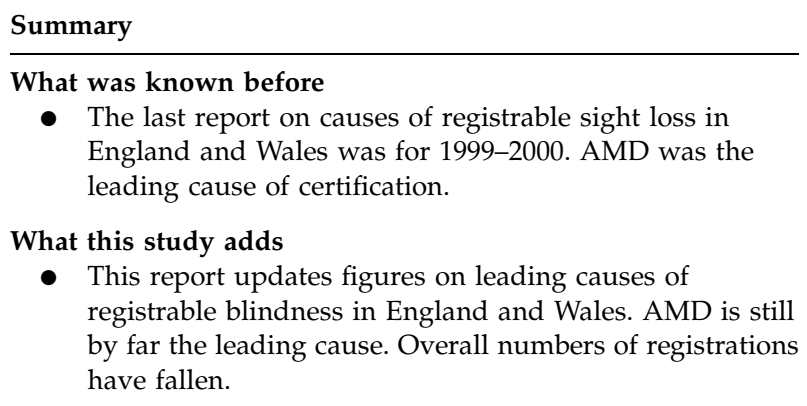

\section{Conflict of interest}

The authors declare no conflict of interest.

\section{Acknowledgements}

This study was supported by a grant from the Guide Dogs and R \& D central funding. The data captured by the CVI are DH copyright and this work was made possible by collaboration with the Royal College of Ophthalmologists. 
The views expressed in this paper are those of the author and not necessarily any funding body or the Department of Health.

\section{References}

1 Sorsby A. The Causes of Blindness in England 1948-50. HMSO: London, 1953.

2 Sorsby A. Blindness in England 1951-54. HMSO: London, 1956.

3 Sorsby A. The Incidence and Causes of Blindness in England and Wales 1948-62. Reports of Public Health and Medical Subjects, Vol 114. HMSO: London, 1966.

4 Sorsby A. The incidence and causes of blindness in England and Wales 1963-8. Reports of Public Health and Medical Subjects, Vol 128. HMSO: London, 1972.

5 Department of Health and Social Security. Blindness and Partial Sight in England 1969-76. Reports of Public Health and Medical Subjects, Vol 129. HMSO: London, 1979.

6 Government Statistical Service. Causes of Blindness and Partial Sight Among Adults in 1976-77 and 1980/81. HMSO: London, 1988.

7 Evans J. Causes of Blindness and Partial Sight in England and Wales 1990-1991. In Studies on Medical and Population Subjects, Vol 57. HMSO: London, 1988.
8 Bunce C, Wormald R. Leading causes of certification for blindness and partial sight in England \& Wales. BMC Public Health 2006; 6: 58.

9 Bunce C, Wormald R. Causes of blind certifications in England and Wales: April 1999-March 2000. Eye (London) 2008; 22(7): 905-911.

10 International Classification of Diseases, Ninth Revision. WHO: Geneva, 1977.

11 Registered Blind and Partially Sighted People Year ending 31 March 2008 England. Information Centre, Adult Social Care Statistics. Available at: http:// www.ic.nhs.uk/pubs/blindpartiallysighted08.

12 Bruce IW, McKennell AC, Walker EC. Blind and Partially Sighted Adults in Britain: The RNIB Survey, Vol 1. HMSO: London, 1991.

13 Walker EC, Tobin MHJ, McKennell AC. Blind and Partially Sighted Children in Britain: The RNIB Survey, Vol 2. HMSO: London, 1992.

14 Robinson R, Deutsch J, Jones HS, Youngson-Reilly S, Hamlin $\mathrm{DM}$, Dhurjon L et al. Unrecognised and unregistered visual impairment. Br J Ophthalmol 1994; 78(10): 736-740.

15 Bunce C, Evans J, Fraser S, Wormald R. BD8 certification of visually impaired people. Br J Ophthalmol 1998; 82(1): 72-76.

16 King AJ, Reddy A, Thompson JR, Rosenthal AR. The rates of blindness and of partial sight registration in glaucoma patients. Eye 2000; 14(Part 4): 613-619.

17 Barry RJ, Murray PI. Unregistered visual impairment: is registration a failing system? Br J Ophthalmol 2005; 89(8): 995-998. 